\title{
Bifurcations and Chaos in Time Delayed Piecewise Linear Dynamical Systems
}

\author{
D. V. Senthilkumar and M. Lakshmanan \\ Centre for Nonlinear Dynamics, Department of Physics, \\ Bharathidasan University, Tiruchirappalli - 620 024, India
}

\begin{abstract}
We reinvestigate the dynamical behavior of a first order scalar nonlinear delay differential equation with piecewise linearity and identify several interesting features in the nature of bifurcations and chaos associated with it as a function of the delay time and external forcing parameters. In particular, we point out that the fixed point solution exhibits a stability island in the two parameter space of time delay and strength of nonlinearity. Significant role played by transients in attaining steady state solutions is pointed out. Various routes to chaos and existence of hyperchaos even for low values of time delay which is evidenced by multiple positive Lyapunov exponents are brought out. The study is extended to the case of two coupled systems, one with delay and the other one without delay.
\end{abstract}




\section{Introduction}

Chaotic phenomenon has been studied extensively in several dynamical systems, including Chua's circuits, cellular neural networks (CNNs) and cellular neural networks with delay (DCNNs) during the past few decades. Dynamical systems described by delay differencedifferential equations have many practical applications in areas such as biology, secure communication, economics, traffic control, neural networks, etc. For example, Mackey and Glass[1977] have demonstrated a model for blood production in patients with leukemia in terms of a delay differential equation, where the concentration of blood can vary chaoti-

cally when request for more blood is made. Time delayed dynamical systems can also play a significant role in secure communications as these systems can have chaotic attractors with a large number of positive Lyapunov exponents when the delay time of the dynamical system is increased [Goedgebuer et al., 1998] and so messages can be more securely transmitted through chaotic synchronization. Time delayed dynamical systems are essentially infinite dimensional systems, as an infinite set of independent numbers are required to specify an initial condition. Such infinite dimensional systems can also be used to forecast the fluctuation in the share market behavior [Gwynne, 2001]. Various parameters in the traffic control theory are also expressed in terms of delay coordinates [Helbing, 2001]. The dynamics of delayed systems depends on the current state as well as on its past history and recursively further past history and so on. Thus CNN's with delay coordinates may be considered as ideal models for neural networks which act with their past knowledge.

It is a widely accepted fact that chaos can occur in autonomous time continuous nonlin- 
ear systems having order greater than two and in nonautonomous time continuous nonlinear systems with order greater than one. In time discrete systems chaos can occur even in first order invertible maps and in non-invertible maps with order greater than one. Recently Lu and $\mathrm{He}[1996]$ have shown that chaos can occur even in a simple scalar first order delayed nonlinear (piecewise linear) dynamical system with large enough delay. Thangavel et al.[1998] have made preliminary study of the bifurcation scenario and controlling of chaos in first order and coupled DCNNs including the model of Lu and He.

In this paper, we have revisited the first order time delayed system studied by Thangavel et al.[1998] with the addition of a constant external forcing term and studied its dynamics in a large parameter regime of the external force and time delay. In particular, we have identified the novel aspects of existence of a stable island in a two parameter space for the equilibrium solutions in the presence of time delay, the significant role of transients in attaining steady state solution and thereby identifying the existence of familiar routes to chaos and the emergence of hyperchaos even for small values of time delay in a scalar first order piecewise linear delay differential equation for a wide range of parameters, which is confirmed by the existence of multiple positive Lyapunov exponents. The study has also been extended to the case in which the first order scalar time delayed system is coupled to a second scalar system without delay. Again the existence of a stable island for equilibrium points is established. Existence of different bifurcation routes including type III intermittency route is pointed out, where we have also discussed about the transient effects. We have also plotted the two parameter bifurcation diagrams for both the cases to bringout the dynamics in two parameter space. The plan of the paper is as follows. To start with, a 
linear stability analysis is carried out both in the presence and in the absence of time delay

in sec. 2. In sec. 3, we have made a detailed study of the effect of transients in attaining the steady state solution leading to identification of typical bifurcation scenario. One and two parameter bifurcation diagrams for different nonlinear parameter regimes characterized by the piecewise linear function for different values of the parameter $p_{2}$ are studied in detail. We also bringout the hyperchaotic nature of the systems with the existence of multiple positive Lyapunov exponents. Further we have considered the addition of one more cell without delay in sec. 4, resulting in two cell DCNN and the results of this system are discussed in sec. 5. The results are summarized in sec. 6 .

\section{Single cell Delayed Cellular Neural Network}

We consider the following first order delay differential equation introduced by Lu and He[1996], but with the addition of a constant external force as studied by Thangavel et al. [1998],

$$
\frac{d x(t)}{d t}=-a x(t)+b f(x(t-\tau))+c,
$$


where $a$ and $b$ are parameters, $\tau$ is the time delay, $c$ is a constant external force and $f$ is an odd piecewise function defined as

$$
f(x(\tau))=\left\{\begin{array}{cc}
0, & x \leq-p_{2} \\
-1.5 x-2, & -p_{2}<x \leq-p_{1} \\
x, & -p_{1}<x \leq p_{1} \\
-1.5 x+2, & p_{1}<x \leq p_{2} \\
0, & x>p_{2} .
\end{array}\right.
$$

Here $p_{1}$ and $p_{2}$ are parameters. The form of the function $f(x(\tau))$ is sketched in Fig. [1 In our study we have fixed the parameter $p_{1}$ at $p_{1}=0.8$ and studied the system behavior in a region of the external force $c \in[-0.15,0.15]$ and time delay $\tau \in[0.0,30.0]$ for different values of the parameter $p_{2}$ characterizing the piecewise linear function $f(x(\tau))$. As any time delayed system depends strongly on its past history, which acts with its rich past knowledge, this model may be viewed as a simple one cell delayed cellular neural network (DCNN). To start with let us consider the nature of the fixed (equilibrium) points of the system (1) in some detail.

\subsection{Fixed points and linear stability}

Equation (1) with the form (2) for $f(x(\tau))$ can admit upto a maximum of three fixed points, $x_{i}(t)=x_{0}, i=1,2,3$, depending upon the parameter values. A linear stability analysis of these fixed points under the linear perturbation $x=x^{*}+\alpha \exp (\lambda t), \alpha \ll 1$, for 
Eq. (1) was carried out in [Thangavel et al., 1998]. However, the stability analysis in the presence of time delay was done very briefly. In this section, we bring out the existence of stable island in the $(\tau, 1.5 b)$ plane for some of these equilibrium points in the presence of time delay in a detailed manner by following the analysis of Ramana Reddy et al. [2000] for the case of coupled limit cycle oscillators with time delay coupling/time delay feedback. For this purpose we examine the stability nature of the fixed points for Eq. (1) both in the absence and in the presence of time delay $\tau$ by considering the characteristic equation for the eigenvalue $\lambda$ and identifying the cases where $\operatorname{Re}(\lambda)<0$ for stability. We consider two cases.

\subsubsection{Time delay $\tau=0$}

In the absence of time delay, the following fixed points can exist depending on the choice of parameters $a, b, c, p_{1}$ and $p_{2}$ in Eqs. (1)-(2):

a) For $|x|>p_{2}$, the fixed point is $x=x_{0}=\frac{c}{a}$ and the characteristic equation in this region is $\lambda=-a$. The fixed point $x=x_{0}=\frac{c}{a}$ is stable for positive values of $a$.

b) For $-p_{2}<x \leq-p_{1}$, the fixed point is $x=x_{0}=\frac{c-2 b}{a+1.5 b}$ and the characteristic equation in this region becomes $\lambda=-(a+1.5 b)$. The fixed point is stable when $a>-1.5 b$.

c) For $|x| \leq p_{1}$, the fixed point is $x=x_{0}=\frac{c}{a-b}$ and the characteristic equation becomes $\lambda=-(a+b)$. The above fixed point is stable when $a>-b$.

d) For $p_{1}<x \leq p_{2}$, the fixed point is $x=x_{0}=\frac{c+2 b}{a+1.5 b}$ and the characteristic equation in this region becomes $\lambda=-(a+1.5 b)$. The fixed point is stable when $a>-1.5 b$.

Next we consider the case when time delay is present, $\tau>0$. 


\subsubsection{Time delay $\tau>0$}

a)Here again, for $|x|>p_{2}$, the fixed point remains the same as for the case $\tau=0$, namely $x=x_{0}=\frac{c}{a}$ and it is stable for $a>0$.

b) Next in the region, $-p_{2}<x \leq-p_{1}$, for the fixed point $x=x_{0}=\frac{c-2 b}{a+1.5 b}$, the characteristic equation becomes the transcendental equation,

$$
\lambda+a+1.5 b e^{-\lambda \tau}=0 .
$$

Let $\lambda=\alpha+i \beta$, where $\alpha$ and $\beta$ are real. Substituting this into the above equation and equating real and imaginary parts, we obtain equations for $\alpha$ and $\beta$ as

$$
\alpha+a+1.5 b e^{-\alpha \tau} \cos (\beta \tau)=0
$$

$$
\beta-1.5 b e^{-\alpha \tau} \sin (\beta \tau)=0
$$

From the above one can easily check by squaring and adding Eqs. (4) and (5) that

$$
\beta=\beta_{ \pm}= \pm \sqrt{2.25 b^{2} \exp (2 \alpha \tau)-(a+\alpha)^{2}}
$$

and

$$
\alpha=-a-\frac{\beta}{\tan (\beta \tau)}
$$


Without loss of generality we choose + sign in the above equation, as the eigenvalues occur in complex conjugate pairs. In order to find the critical stability curves, we choose $\alpha=0$. Then we have

$$
\left.\beta\right|_{\alpha=0}= \pm \sqrt{2.25 b^{2}-a^{2}} .
$$

From Eq. (4), it follows that

$$
\beta \tau= \pm \cos ^{-1}\left(\frac{-a}{1.5 b}\right)+2 n \pi,
$$

where $\mathrm{n}$ is any integer $(0, \pm 1, \pm 2, \ldots)$. Consequently one can expect that the stability regions are confined between the set of two curves

$$
\begin{aligned}
\tau_{1}(n, 1.5 b) & =\frac{2 n \pi+\cos ^{-1}\left(\frac{-a}{1.5 b}\right)}{\sqrt{2.25 b^{2}-a^{2}}}, \\
\tau_{2}(n, 1.5 b) & =\frac{2 n \pi-\cos ^{-1}\left(\frac{-a}{1.5 b}\right)}{\sqrt{2.25 b^{2}-a^{2}}} .
\end{aligned}
$$

In Eq. (10a), $n=0,1,2, \ldots$ and in Eq. (10b), $n=1,2, \ldots$ for the pair of curves we have chosen so that the curves have positive value of $\tau$. To start with we note from Eq. (3) that when the time delay $\tau=0, \lambda=-a-1.5 b$ and so $\alpha<0$. In order to identify those curves for $\tau>0$ which encompass the stable regions, the critical curves should be the ones on which $\frac{d \lambda}{d \tau}>0$. From Eq. (3),

$$
\frac{d \lambda}{d \tau}=\frac{1.5 b \lambda \exp (-\lambda \tau)}{1-\tau 1.5 b \exp (-\lambda \tau)}
$$


and hence

$$
\begin{aligned}
\left.\frac{d \alpha}{d \tau}\right|_{\alpha=0} & =\operatorname{Re} \frac{1.5 b(i \beta) \exp (-i \beta \tau)}{1-\tau 1.5 b \exp (-i \beta \tau)} \\
& =\frac{1.5 b \beta \sin (\beta \tau)}{D} \\
& =\beta^{2} D^{-1}
\end{aligned}
$$

where

$$
D=[1-1.5 b \tau \cos (\beta \tau)]^{2}+[1.5 b \tau \cos (\beta \tau)]^{2} .
$$

Therefore

$$
\frac{d \alpha}{d \tau}>0 \text { on both } \tau_{1} \text { and } \tau_{2}
$$

The above condition implies that there can be only one stability region between $\tau=0$ line (where $\alpha<0)$ and the critical curve $\tau_{1}(0,1.5 b)$ which is the closest to the line $\tau=0$. We note that the condition (13) prohibits the existence of any other stable region (that is multistability regions) as in the case of time delayed limit cycle oscillators [Ramana Reddy et al., 1998; 2000], because for a second stable region to exist one requires $\frac{d \alpha}{d \tau}<0$ on any one of the other curves $(n>1)$. But this never occurs in our case. The numerical plot in Fig. 2 of the curves $\tau_{1}(n, 1.5 b)$ (solid curve for $\left.n=0,1,2\right)$ and $\tau_{2}(n, 1.5 b)$ (broken curve for $n=1,2)$ reveals that the region between $\tau=0$ and $\tau=\tau_{1}(0,1.5 b)$ is the only stable region (shaded region), where $\frac{d \alpha}{d \tau}$ on $\tau_{1}>0$, which passes from negative to positive values of $\alpha$, whereas the other curves $\tau_{2}(n, 1.5 b)<\tau<\tau_{1}(n, 1.5 b)$ for $n>0$ do not satisfy the required stability condition and hence they are all associated with unstable regions. 
c) As in the previous region, for the case $-p_{1}<x \leq p_{1}$ and for the fixed point $x_{0}=\frac{c}{a-b}$, the characteristic equation in this region has the form

$$
\lambda+a+b e^{-\lambda \tau}=0
$$

As before, we obtain a set of critical curves as

$$
\begin{aligned}
& \tau_{1}(n, b)=\frac{2 n \pi+\cos ^{-1}\left(\frac{a}{b}\right)}{\sqrt{b^{2}-a^{2}}}, \\
& \tau_{2}(n, b)=\frac{2 n \pi-\cos ^{-1}\left(\frac{a}{b}\right)}{\sqrt{b^{2}-a^{2}}},
\end{aligned}
$$

where $n=0,+1,+2, \ldots$ in Eq. (15a) and $n=+1,+2, \ldots$ in Eq. (15b). Also we get

$$
\left.\frac{d \alpha}{d \tau}\right|_{\alpha=0}=\beta^{2} D^{-1}
$$

where

$$
\begin{aligned}
D & =[1-1.5 b \tau \cos (\beta \tau)]^{2}+[1.5 b \tau \cos (\beta \tau)]^{2} \\
\beta & =b \sin (\beta \tau)
\end{aligned}
$$

with the same argument as for the above case, we find that there is only one stable island between the curves $\tau=0$ and $\tau_{1}(0, b)$.

d)For the case $p_{1}<x \leq p_{2}$ and for the fixed point $x=x_{0}=\frac{c+2 b}{a+1.5 b}$, the characteristic equation turns out to be identical to Eq. (3) and the associated critical curves also have 
similar form as that of Eq. (10).

\section{$3 \quad$ Numerical study of the single cell DCNN}

In this section, we will present a discussion of the dynamics of the delayed cellular neural network (Eq. (1)) in the pseudospace $(x(t), x(t-\tau))$. Further we will also discuss the significant role played by transients in attaining the steady state behavior, the computational efficiency required for achieving such steady state solution and the nature of bifurcation diagrams for both low and high transients with the external forcing $c$ as the control parameter. We will also point out the existence of a stable island for equilibrium points in the two parameter (now in the time delay $\tau$ - the external forcing $c$ plane) bifurcation diagrams for various ranges of control parameters and nonlinearity characterized by the function $f(x(\tau))$ for three different values of $p_{2}$ in Eq. (2). In addition, we have also calculated the Lyapunov exponents associated with the system and show that there exists a large parameter range over which hyperchaos exists (corresponding to multiple positive Lyapunov exponents). From the two parameter bifurcation diagrams, it becomes evident that the sizes of the hyperchaotic regime and the stable fixed point regime increase with time delay.

\subsection{Dynamics in the pseudospace}

The dynamics of the DCNN can be studied in a suitable phase space by plotting the numerical solution of Eq. (1) appropriately. Now, to calculate $x(t)$ from Eq. (1) for times greater 
than $t$, the function $x(t)$ over the interval $(t-\tau, t)$ must be given. Hence, for a prescribed continuous function $x(t)$ on $(-\tau, 0)$ one can integrate Eq. (1) using numerical methods as in the case of ordinary differential equations. We have numerically integrated Eq. (1) using Runge-Kutta fourth order integration routine with the parameters $a=1.0, b=1.2$ and $p_{1}=0.8$ for three different values of $p_{2}=1.0,1.33,1.66$ with the initial condition $x(t)=0.9$ in the interval $(-\tau, 0)$. As the system is of first order in nature, the dynamics can be viewed in a pseudospace by plotting $x(t)$ against $x(t-\tau)$. (Note that the choice of $x(t-\tau)$ as the second phase variable is arbitrary; $x\left(t-t^{\prime}\right)$ can be equivalently used, where $t^{\prime}$ is an arbitrary time delay). One encounters typical scenario of bifurcations leading to chaos, but with an important difference: Transients play a crucial role and it takes a very long time before transients die down to attain steady state solutions. In particular, period doubling and inverse period doubling phenomena, besides other bifurcations, are often encountered. In many cases the system exhibits hyperchaotic behavior characterized by multiple positive Lyapunov exponents, which we have discussed below in sec. 3.4. Fig. 3 shows a typical pseudochaotic attractor for $\tau=5.0, c=0.001$ and $p_{2}=1.33$ with the initial condition as given above in the phase space of $x(t)$ against $x(t-5.0)$. The positive maximal Lyapunov exponent of this attractor for the above mentioned parameter values is $\lambda_{\max }=0.05461$. It may be noted that previously it was reported by Thangavel et al. [1998] and Lu and He [1996] that the system for a different set of parameters exhibits only chaos (and not hyperchaos) for large values of time delays, that is $\tau>20$. On the otherhand we have identified the parameter regimes where the system exhibits chaos and even hyperchaos for small values of time delay around $\tau=5.0$. In the following we present the details. 


\subsection{Transients}

Usually dynamical systems described by ordinary differential equations can attain the steady state within a few thousand transients for optimal value of time step $\Delta t$, provided due care is given for numerical accuracy and suitable numerical algorithm is chosen. However for the delay differential equation considered in this paper, such low transients (of the order $10^{4}$ and more) do not seem to lead the system to their steady state solutions. In such cases one has to leave out more number of transients so that one can have the possibility of obtaining a clear picture of the bifurcation diagram with the existing routes. In fact, the effect of transients has been pointed out by Becker and Dorfler[1989] even for the logistic map. As for as nonlinear maps and ordinary differential equations are concerned, with some effort it is possible to realize whether the system has reached the steady state or not by constructing the appropriate bifurcation diagrams and identifying the various bifurcation routes by leaving sufficient number of transients before starting the analysis for steady state solutions. The same considerations hold good for dynamical systems modeled by finite number of coupled nonlinear maps and odes without time delay.

On the other hand, when one starts studying nonlinear dynamical systems with time delay, effectively one considers infinite number of coupled odes which in a numerical sense corresponds to several hundred coupled nonlinear odes. For example, for Eq. (1), when the time delay $\tau=25.0$, in the actual numerical analysis, we typically take the optimal time step as $\Delta t=0.05$, so that we are actually solving 500 coupled nonlinear odes. However, the optimal time step $\Delta t$ to be fixed depends explicitly on the nature of the system and on the 
time delay introduced in the system. In contrast to the nonlinear odes, where either a very small or a relatively large time step $\Delta t$ results in an exponentially increasing numerical error, for the time delayed system (1), we have found that the optimal time step $\Delta t$ varies between 0.05 to 0.0001 , depending on which the actual number of coupled nonlinear odes increase, and the number of transients to be left out is found to lie anywhere between $2.0-3.0 \times 10^{6}$. For small values of time delay $\tau$, the optimal time step $\Delta t$ should be as small as possible to identify a typical bifurcation scenario. However, for large values of time delay $\tau$, a very small value of time step $\Delta t$ can again be problematic as one has to deal with a large number of coupled odes and the time required for solving such huge number of coupled odes becomes huge. Also a large value of time step $\Delta t$ does not seem to lead to the steady state solution, as such time steps lead to propagation of numerical errors. However, an appropriate optimal choice of time step $\Delta t$ can lead to a typical bifurcation scenario.

Thus the numerical analysis of time delayed nonlinear systems requires an appropriate time step $\Delta t$ to be chosen, depending on which the actual number of coupled nonlinear odes increases, which in turn necessarily increases the number of transients to be left out in order to obtain the steady state solution. In such cases computing with a standard personal computer like a 32bit Pentium IV processor is a difficult and time consuming task. However, it turns out that with a high speed computers like an Alpha workstation, such a task becomes feasible. We have carried out our analysis with a 64 bit Alpha workstation, in which it took 50-60 days to plot each of the two parameter bifurcation diagrams leaving out sufficient transients using Runge-Kutta fourth order integration routine. 
In our numerical analysis we have chosen the optimal time step as $\Delta t=0.05$ for the value of time delay $\tau=25.0$. The effect of transients in reaching the steady state behavior can be realized from the bifurcation diagrams we have obtained for the parameter values $a=0.16, b=0.2$ and for two different values of $p_{2}$. Fig. 椟 shows the one parameter bifurcation diagram for the above mentioned parameter values with $p_{2}=1.33$, when we leave transients of the order $1.0-2.0 \times 10^{4}$, which is sufficiently large in the case of nonlinear maps and odes, leaving the impression that the steady state has been reached with a bifurcation scenario that is quite complicated and atypical. In contrast, Fig. 枯 shows the bifurcation diagram for the same value of the parameters except that now the transients left out are of the order of $1.0 \times 10^{5}$, which shows a typical bifurcation scenario. Similarly Fig. 5 a shows the bifurcation diagram for the same values of parameters as above for $\tau, a$ and $b$ with $p_{2}=1.66$ for the transients of the order $1.0 \times 10^{5}$, which shows a very complex structure, whereas Fig. [5 shows the bifurcation diagram for the transients of the order $1.4 \times 10^{6}$, in which case still there exists some stray points near the bifurcation regions, which indicates that the system requires still more transients to settle to its steady state. The complexity increases with time delay and hence the number of transients also increases, which in turn increases the computing time enormously. This is also evident from Fig. [6, where the maximum value of $x(t)$, XMAX, is plotted against the time delay $\tau$, for the parameter values $a=1.0, b=1.2, c=0.001$ and $p_{2}=1.33$. Fig. [6 bhows that even for transients of the order $1.0 \times 10^{5}$, the bifurcation diagram is still in an unsettled form for larger values of time delay $\tau$. On the other hand in Fig. [b, the number of transients is of the order $2.5 \times 10^{5}$, wherein a clear bifurcation scenario has emerged. We have verified the 
role of transients to attain the steady state solutions for various values of time step $\Delta t$ and time delay $\tau$ for few other delay differential equations also, including those discussed in the work of Ramana Reddy et al., [2002]. The results of our detailed numerical investigation for Eq. (1) are tabulated in Table I, where we have indicated the number of transients to be left out for given values of $\Delta t$ in order to attain the steady state solutions.

Table I: Effect of step-size on transients

\begin{tabular}{|c|c|c|c|}
\hline Value of $\tau$ & Optimal value of $\Delta t$ & $\begin{array}{c}\text { Number of coupled } \\
\text { differential equations }\end{array}$ & $\begin{array}{c}\text { Number of transients } \\
\text { to be left out }\end{array}$ \\
\hline 5 & 0.002 & 250 & $3.0-5.0 \times 10^{5}$ \\
& 0.001 & 500 & $5.0-6.0 \times 10^{5}$ \\
10 & 0.002 & 5000 & $1.0-2.0 \times 10^{6}$ \\
25 & 0.001 & 10000 & $>2.0 \times 10^{6}$ \\
& 0.05 & 500 & $1.0 \times 10^{5}-2.0 \times 10^{6}$ \\
& 0.005 & 5000 & $1.0-2.0 \times 10^{6}$ \\
& 0.0025 & 10000 & $>2.0 \times 10^{6}$ \\
\hline
\end{tabular}

As the time delay increases, the size of the delay loop (that is to be updated on every iteration) required to maintain the delay variable in numerical simulation also increases, which in turn increases the computational effort. Another important point to note in connection with the effect of transients is the following. In the above we have discussed the effect with reference to a scalar delay differential equation only. On the other hand, in recent times, there has been considerable interest to study the dynamics of multiply coupled delayed neural networks in biological systems [Campbell et al., 1999; Earl \& Strogatz, 2003]. 
In order to examine the actual steady state behavior of such networks, which effectively corresponds to several thousands of coupled nonlinear odes, the time required to obtain steady state solution increases enormously as each one of them have separate time delay and intrinsic transient behavior and it becomes crucial to study the effect of the later.

\subsection{One and two parameter bifurcation diagrams}

All the bifurcation diagrams shown in this paper are plotted after leaving out a very large number transients of of the order of $1.2-3.2 \times 10^{6}$. Fig. 团 shows the one parameter (c) bifurcation diagram characterized by the piecewise linear function $f(x)$ in Eq. (2) with $p_{1}=0.8, p_{2}=4 / 3, \tau=25.0$ for $c \in[-0.1,-0.05]$. It clearly exhibits period doubling scenario; however there exists a sudden distortion around the value of $c=-0.0785$, the cause of which remains unexplained by any standard type of bifurcation. (We wish to point out that the numerical analysis in [Thangavel et al., 1998] actually corresponds to the value of $p_{2}=1.0$ and not $p_{2}=4 / 3$ as stated in that paper). Similarly Fig. 5 b shows the bifurcation diagram with the values $p_{1}=0.8, p_{2}=5 / 3, \tau=25.0$ within the range of $c \in[-0.15,-0.136]$, exhibiting reverse period doubling route to chaos in the range of $c \in[-0.136,-0.144]$ that includes a clear band merging crises and anti-monotonicity in the range of $c \in[-0.15,-0.144]$.

The two parameter bifurcation diagram for $\tau \in[0,30]$ and $c \in[-0.16,0.16]$ when $p_{2}=1.0$ is shown in Fig. $7 \mathrm{t}$, which shows the behavior of the DCNN for the combined phase space of parameters $\tau$ and $c$. The following colour codes are used to represent 
various regions, period-1 region -red, period-2 region - green, 3-blue, 4-yellow, 5-magenta, 6-cyan, 7-gray, 8-copper, chaos-black and the fixed points-white. The white region in the two parameter bifurcation diagram corresponds to stable regions. Fig. $7 \mathrm{~b}$ shows the two parameter bifurcation diagram for the same parameters as in Fig. [ $7 \mathrm{a}$, except that now $p_{2}=4 / 3$. We use the same colour codes as in Fig. 7 f for all the two parameter bifurcation diagrams in this paper. Similarly Fig. 7 r shows the two parameter bifurcation diagram for the same range of the control parameters and the same values of the parameters $a$ and $b$ in Eq. (1) except that now $p_{2}=5 / 3$. By comparing the Figs. $7 \mathrm{~b}, \mathbf{7 b}$ and $7 \mathrm{f}$, we can see that the stable fixed point regions and the chaotic regions increase for small changes in the nonlinear parameters $p_{1}$ and $p_{2}$. Further we infer from these figures that as the delay increases the chaotic nature of the system also increases and thereby contributing to the hyperchaotic nature of the system characterized by multiple positive Lyapunov exponents.

\subsection{Lyapunov exponents and hyperchaotic regimes}

One of the interesting aspects of the dynamics associated with Eq. (1) is the existence of hyperchaos in a single first order scalar equation with time delay even for small values of the latter for suitable values of other system parameters, whereas mention was made in the earlier papers [Lu \& He, 1996; Thangavel et al., 1998] that the system Eq. (1) exhibits only simple chaos even for large values of time delay. As Eq. (1) is a first order delay differential equation, the usual procedure for calculating Lyapunov exponents is not applicable. However, the simple idea of approximating a single scalar differential 
equation with delay by an N-dimensional discrete mapping [Farmer; 1982], which is of infinite dimensions, facilitates one to calculate the Lyapunov exponents. To stimulate the behavior of such systems on a computer it is necessary to approximate the continuous evolution of an infinite dimensional system by a finite number of elements whose values change at discrete time steps. In this manner a continuous infinite dimensional dynamical system is replaced by a finite dimensional iterated mapping. This method was proposed originally by Farmer[1982] to calculate the Lyapunov exponents for delay systems. As the delay parameter is increased, for most parameter values the dimension increases and the attractor generally becomes more complicated, thereby contributing to the hyperchaotic nature of the system, which gets confirmed by the increasing number of positive Lyapunov exponents. The first ten maximal Lyapunov exponents, for the parameter values $a=$ $1.0, b=1.2, c=0.001, p_{1}=0.8, p_{2}=1.33$ and $\tau \in(2,29)$ is shown in Fig. 8, where it is evident that the number of positive Lyapunov exponents increases with time delay $\tau$. Almost the entire black regime in the two parameter bifurcation diagrams Fig. 7 for large values of $\tau$ is characterized by multiple positive Lyapunov exponents contributing to hyperchaotic nature of the system. 


\section{Stability analysis and chaotic dynamics of two cell DCNN}

We now add one new cell without delay to the Eq. (1) to obtain the following coupled equation

$$
\begin{aligned}
& \frac{d x(t)}{d t}=-a x(t)+b f(x(t-\tau))+d y(t), \\
& \frac{d y(t)}{d t}=-c y(t)+e x(t),
\end{aligned}
$$

where $c$ and $e$ are additional parameters and the function $f(x)$ and other parameters are the same as already defined in Eq. (1). As in the case of single cell DCNN, we have fixed the parameter $p_{1}$ at $p_{1}=0.8$ and studied the system behavior in a range of variable parameter $c \in[0.0,1.4]$ and time delay $\tau \in[0.0,30.0]$ for different values of the parameter $p_{2}$ characterizing the piecewise linear function $f(x)$. Eq. (16) can also be viewed as a two cell DCNN, one cell acting with its past knowledge coupled with another cell which does not have any past knowledge. Now let us consider the nature of the fixed points of system (16).

\subsection{Fixed points and Linear stability}

We will bring out the existence of stable island in two cell DCNN by linear stability analysis in the presence of time delay $\tau$, as in the case of single cell DCNN. For this purpose, we examine the stability nature of the fixed points of Eq. (16) both in the absence and in the 
presence of time delay $\tau$.

\subsubsection{Time delay $\tau=0$}

In the absence of time delay, the following fixed points can exist depending on the choice of parameters $a, b, c, d, e, p_{1}$ and $p_{2}$ in Eqs. (16) and (2).

a) For $|x|>p_{2}$, the fixed point is $(x, y)=\left(x_{0}, y_{0}\right)=(0,0)$ and the characteristic equation in this region is $\lambda^{2}+(a+c) \lambda+a c-d e=0$. The fixed point $(x, y)=(0,0)$ is stable when $a+c>0$ and $a c>d e$.

b) For $-p_{2}<x \leq-p_{1}$, the fixed point is $(x, y)=\left(\frac{2 b c}{d e-(a+1.5 b) c}, \frac{2 b e}{d e-(a+1.5 b) c}\right)$ and the characteristic equation in this region becomes $\lambda^{2}+(c+a+1.5 b) \lambda+(c a+1.5 b c-d e)=0$. The fixed point is stable when $c+a+1.5 b>0$ and $a c+1.5 b c>d e$.

c) For $|x| \leq p_{1}$, the fixed point is $(x, y)=(0,0)$ and the characteristic equation becomes $\lambda^{2}+(c+a-b) \lambda+(c a-b c-d e)=0$. The above fixed point is stable when $c+a-b>0$ and $a c>b c+d e$.

d) For $p_{1}<x \leq p_{2}$, the fixed point is $(x, y)=\left(\frac{-2 b c}{d e-(a+1.5 b) c}, \frac{-2 b e}{d e-(a+1.5 b) c}\right)$ and the characteristic equation in this region becomes $\lambda^{2}+(c+a+1.5 b) \lambda+(c a+1.5 b c-d e)=0$. The fixed point is stable when $c+a+1.5 b>0$ and $a c+1.5 b c>d e$.

Next we consider the case when time delay is present, $\tau>0$.

\subsubsection{Time delay $\tau>0$}

a) For $|x|>p_{2}$, the fixed point and its stability remains the same as for the case $\tau=0.0$

b) Next in the region $-p_{2}<x \leq-p_{1}$, for the fixed point $(x, y)=\left(\frac{2 b c}{d e-(a+1.5 b) c}, \frac{2 b e}{d e-(a+1.5 b) c}\right)$ 
the characteristic equation is

$$
(c+\lambda)(\lambda+a+1.5 b \exp (-\lambda \tau))-d e=0,
$$

which is a transcendental equation with infinite number of solutions. Let $\lambda=\alpha+i \beta$, where $\alpha$ and $\beta$ are real. Substituting this in the above equation and equating real and imaginary parts, we obtain two equations as

$$
\begin{array}{r}
(c+\alpha)(\alpha+a+1.5 b \exp (-\alpha \tau) \cos (\beta \tau))-d e-\beta^{2}+1.5 \beta b \exp (-\alpha \tau) \sin (\beta \tau)=0 \\
(c+\alpha)(\beta-1.5 b \exp (-\alpha \tau) \sin (\beta \tau))+\beta(\alpha+a+1.5 b \exp (-\alpha \tau) \cos (\beta \tau))=0
\end{array}
$$

In order to find the critical stability curves, we choose $\alpha=0$. Then we have

$$
\begin{array}{r}
1.5 b c \cos (\beta \tau)+c a-d e-\beta^{2}+1.5 \beta b \sin (\beta \tau)=0 \\
1.5 b \beta \cos (\beta \tau)+c \beta+\beta a-1.5 b c \sin (\beta \tau)=0 .
\end{array}
$$

Multiplying the above two equations (19a) and (19b) with $c$ and $\beta$, respectively, adding we obtain

$$
1.5 b \cos (\beta \tau)\left(c^{2}+\beta^{2}\right)+a\left(c^{2}+\beta^{2}\right)-c d e=0 .
$$


Now multiplying the equations (19a) and (19b) with $\beta$ and $c$, respectively, subtracting the resulting equations, we obtain

$$
1.5 b \sin (\beta \tau)\left(c^{2}+\beta^{2}\right)-\beta\left(c^{2}+\beta^{2}\right)-\beta d e=0 .
$$

Squaring and adding the equations (201) and (21), rearranging them, we obtain the following cubic equation for $\beta^{2}$,

$$
X^{3}+u X^{2}+v X+w=0, X=\beta^{2},
$$

where the constants are given as

$u=a^{2}+2\left(d e+c^{2}\right)-2.25 b^{2} ; v=\left(d e+c^{2}\right)^{2}-2 a c(d e-a c)-4.5 b^{2} c^{2} ; w=c^{2}(d e-a c)^{2}-2.25 b^{2} c^{4}$.

From Eq. (201), we obtain

$$
\beta \tau= \pm \cos ^{-1}\left(\frac{c d e-a\left(c^{2}+\beta^{2}\right)}{1.5 b\left(c^{2}+\beta^{2}\right)}\right)+2 n \pi
$$

where $n$ is any integer $(0, \pm 1, \pm 2, \ldots)$ and the value of $\beta$ can be obtained by solving the Eq. (22) for $\beta^{2}$. Consequently the stability regions are confined between the set of curves,

$$
\begin{aligned}
\tau_{1}(n, 1.5 b) & =\frac{2 n \pi+\cos ^{-1}\left(\frac{c d e-a\left(c^{2}+\beta^{2}\right)}{1.5 b\left(c^{2}+\beta^{2}\right)}\right)}{\beta} \\
\tau_{2}(n, 1.5 b) & =\frac{2 n \pi-\cos ^{-1}\left(\frac{c d e-a\left(c^{2}+\beta^{2}\right)}{1.5 b\left(c^{2}+\beta^{2}\right)}\right)}{\beta},
\end{aligned}
$$


where $n=0,+1,+2, \ldots$ in Eq. (24a) and $n=+1,+2, \ldots$ in Eq. (24b). In order to check whether the region enclosed by the curve $\tau_{1}$ and $\tau_{2}$ for every value of $n$ forms stable islands in $(\tau, \beta)$ plane, one has to examine the sign of $\frac{d \alpha}{d \tau}$ on $\tau_{1}$ and $\tau_{2}$ as we have done for the case of single cell DCNN. We have found that there exists only one stable island between $\tau=0$ and $\tau=\tau_{1}(0, \beta)$ curve (shaded region) as shown in Fig. 9 .

c) In the region $|x| \leq p_{1}$, for the fixed point $(x, y)=(0,0)$, the characteristic equation is

$$
(c+\lambda)(\lambda+a+b \exp (-\lambda \tau))-d e=0,
$$

is a transcendental equation with infinite number of solutions. Proceeding in the same way as for the case $-p_{2}<x \leq-p_{1}$, we obtain a set of critical curves as

$$
\begin{gathered}
\tau_{1}(n, b)=\frac{2 n \pi+\cos ^{-1}\left(\frac{c d e-a\left(c^{2}+\beta^{2}\right)}{b\left(c^{2}+\beta^{2}\right)}\right)}{\beta} \\
\tau_{2}(n, b)=\frac{2 n \pi-\cos ^{-1}\left(\frac{c d e-a\left(c^{2}+\beta^{2}\right)}{b\left(c^{2}+\beta^{2}\right)}\right)}{\beta},
\end{gathered}
$$

where $n=0,+1,+2, \ldots$ in Eq. (25a) and $n=+1,+2, \ldots$ in Eq. (25b). As for the previous case we found that there is only one stable regions between $\tau=0$ and $\tau=\tau_{1}(0, \beta)$.

d)For the case $p_{1}<x \leq p_{2}$ and for the fixed point $(x, y)=\left(\frac{-2 b c}{d e-(a+1.5 b) c}, \frac{-2 b e}{d e-(a+1.5 b) c}\right)$, the characteristic equation turns out to be indentical to Eq. (17) and the associated critical curves also have similar form as that of Eqs. (24a) and (24b). 


\section{Numerical study the two cell DCNN}

In this section, we will discuss the dynamics of the two cell DCNN defined by Eq. (16) through numerical analysis and the transient effects involved in it by studying the bifurcation diagrams for both low and high transients with $c$ as control parameter. The one parameter bifurcation diagram in one of the regimes has the signature of type III intermittent behavior. We have also found that the dynamics at this transition possesses type III intermittent characteristic scaling behavior. We have also plotted the two parameter bifurcation diagrams in the $(\tau, c)$ plane for three different values of $p_{2}$, from which also we have pointed out the existence of stable island for equilibrium points for suitable choice of other parameter values.

\subsection{Transients}

As we have already discussed much about the role of transients in the case of single cell DCNN, in Sec. 3.2, it is not necessary to discuss it once again in detail for the present case. So we point out only the number of transients required to obtain the bifurcation diagrams which we have shown in Figs. 10] and 11 for the system(16), which enables one to realize the effect of transients. As discussed in the case of single cell DCNN, the transients predominates evolution of the system(16) to its steady state solutions. Fig. 10a shows the one parameteriii bifurcation diagram for transients of the order $2.0 \times 10^{5}$ for the parameter value $p_{2}=1.33$, whereas Fig. 10] shows the one parameter bifurcation diagram for transients of order $1.75 \times 10^{6}$ for the same parameter values. The stray points in the 
neighbourhood of bifurcation points are due to the transient effects, which suggests that it requires still more iterations need to be left out. Fig. 11 shows the one parameter bifurcation diagram for transients of the order $2.0 \times 10^{5}$ for the parameter value $p_{2}=1.33$, whereas Fig. 11]b shows the bifurcation diagram for transients of the order $2.0 \times 10^{6}$ for the fixed values of other parameters.

\subsection{One and two parameter bifurcation diagrams}

We have integrated Eq. (16) with the parameters $a=0.16, b=0.2, d=0.2, e=0.2$ and $c$ as variable parameter with the initial conditions $x(t)=0.9$ for $t \in(-25,0)$ and $y=0.8$. Fig. 10b shows the one parameter bifurcation diagram in the nonlinear parameter regime characterized by the function $f(x(\tau))$ with $p_{1}=0.8, p_{2}=4 / 3$ and $\tau=25.0$, which shows the period-3 doubling bifurcation route to chaos. Fig. 11] shows reverse period doubling in the range $c \in[0.32,0.345]$, and at the critical value of $c_{c r i t}=0.345969$ the system exhibits intermittent transition to chaos followed by reverse period 5 doubling in the range $c \in[0.346,0.365]$ interspersed by periodic windows for the same parameter values as above except that now $p_{2}=5 / 3$. At the intermittent transition, the amplitude variation loses its regularity and a burst appears in the regular phase as shown in Fig. 12. This behavior repeats as time increases as observed in the usual type III intermittent scenario. The duration of laminar phases is random during the transition and finally results in chaotic oscillations by increasing the critical value of the control parameter $c$. The plot of mean laminar length $\left\langle l>\right.$ as a function of the parameter $f=\left(c_{c r i t}-c\right)$ is shown in Fig. 13, where 
$c_{c r i t}$ is the critical value of the parameter for the occurrence of the intermittent transition. The phase space trajectories reveal a power law relationship of the form $<l>=f^{-\alpha}$ with the estimated value of $\alpha=0.871$. This analysis confirms that the trajectories at the critical value of $c$ is associated with standard intermittent dynamics of type III described in the work of Pomeau \& Manneville [1980] and Schuster [1988]. Figs. 14] shows the global bifurcation diagrams when $p_{2}=1.0,4 / 3$ and $5 / 3$ respectively. The colour codes used here are the same as in the previous case of single cell DCNN. By comparing the global bifurcation diagrams, we can realize that the stable island (fixed point) and the chaotic region increases for small changes (increase) in the parameter $p_{2}$. In addition the chaotic nature of the system increases with delay.

\section{Conclusion}

We have studied in detail the linear stability nature of equilibrium points, both in the absence and in the presence of time delay, of the DCNN systems and identified the existence of stable island in a single scalar piecewise linear differential equation with inherent time delay. The significant role of transients in attaining the steady state behavior has also been brought out. One and two parameter bifurcation diagrams for specific choice of parameters have been presented in support of our results. Hyperchaotic nature of the system, even for small time delay in a simple first order piecewise linear differential equation ensures that such systems can be used for highly efficient secure communication by constructing equivalent electronic circuits. Therefore based on our results the respective circuits with 
suitable delay can be designed and can be tested for specific applications. These studies have also been extended to coupled systems with time delay and the generic nature of the results established.

\section{Acknowledgments}

This work forms part of a Department of Science and Technology, Government of India sponsored research project. 


\section{References}

1. Becker, K. H. \& Dorfler, M. [1989] Dynamical systems and fractals (Cambridge University Press, Cambridge).

2. Campbell, S. A., Ruan, S. \& Wei, J. [1999] "Qualitative Analysis of a Neural Network Model with MultipleTime Delays," Int. J. of Bifurcation and chaos 9(8), 1585-1595.

3. Earl, M. G. \& Strogatz, S. H. [2003] "Synchronization in Oscillator Networks with Delayed Coupling:A Stability Criterion," Phys. Rev. E. 67, 036204.

4. Farmer, J. D. [1982] "Chaotic Attractor of an Infinite-Dimensional Dynamical System," Physica 4D, 366-393.

5. Goedgebuer, J. P., Larger, L. \& Porte, H. [1998] "Optical Cryptosystem Based on Synchronization of Hyperchaos Generated by a Delayed Feedback Tunable Laser Diode," Phys. Rev. Lett. 80, 2249-2252.

6. Gwynne, P. [2001] "Physicist who makes Cash from Chaos," Physics World 9, 9.

7. Helbing, D. [2001] "Traffic and Related Self-driven Many-particle Systems," Rev. of Mod. Phys. 73, 1067-1141.

8. Herrero, R., Figueras, M., Rius, J., Pi, F., \& Orriols, G. [2000] "Experimental Observation of the Amplitude Death Effect in Two Coupled Nonlinear Oscillator," Phys. Rev. Lett. 84, 5312-5316. 
9. Ikeda, K. \& Matsumato, K. [1987] "High-dimensional Chaotic Behavior in Systems with Time Delayed Feedback," Physica D 29, 223-235.

10. Kim, S., Park, S. H., \& Ryu, C. S. [1997] "Multistability in Coupled Oscillator Systems with Time Delay," Phys. Rev. Lett. 79, 2911-2915.

11. Lim, T. K., Kwak, K. \& Yun, M. [1998] "An Experimental Study of Storing Information in a Controlled Chaotic System with Time Delayed Feedback," Phys. Lett. A 240, 287-294.

12. Lu, H. \& He, Z. [1996] "Chaotic Behavior in first Order Autonomous Continuous Time System with Delay," IEEE Trans. Circuits Syst.I 43, 700-702.

13. Mackey, M. C. \& Glass, L. [1977] "Oscillation and Chaos in Physiological Control Systems," Science 197, 287-289.

14. Ott, E. [1993] Chaos in dynamical systems (Cambridge University Press, Cambridge).

15. Pomeau, Y. \& Manneville, P. [1980] "Intermittent Transition to Turbulence in Dissipative System," Commun. Maths. Phys. 74, 189-197.

16. Ramana Reddy, D. V., Sen, A. \& Johnston, G. L. [1998] "Time Delay Induced Death in Coupled Limit Cycle Oscillators," Phys. Rev. Lett. 80, 5109-5113.

17. Ramana Reddy, D. V., Sen, A. \& Johnston, G. L. [2000] "Experimental Evidence of Time Delay Induced Death in Coupled Limit Cycle Oscillators," Phys. Rev. Lett. 85, 3381-3385. 
18. Schuster, H. G. [1988] Deterministic Chaos (VCH Publishers, Weinheim).

19. Thangavel, P., Murali, K. \& Lakshmanan, M. [1998] "Bifurcation and Controlling of Chaotic Delayed Cellular Neural Networks," Int. J. of Bifurcation and chaos 8, 2481-2492.

20. Yaowen, L., Guangming, G., Hong, Z. \& Yinghai, W. [2000] "Synchronization of Hyperchaotic Harmonics in Time Delay Systems and its Application to Secure Communication," Phys. Rev. E. 62, 7898-7904. 
Figure 1: The form of the piecewise linear function $f(x(\tau))$ in Eq. (1) for the values $p_{1}=0.8, p_{2}=1.33$.

Figure 2: Curves of Eq. (10a) and Eq. (10b) in the range of $-p_{1}<x \leq p_{1}$. The solid curve represents $\tau_{1}$ for $n=0,+1,+2$ and broken curve represents $\tau_{2}$ for $n=+1,+2$. The region enclosed between the line $\tau=0$ and the curve $\tau=\tau_{1}(0,1.5 b)$ is the only stable island (shaded region).

Figure 3: Pseudochaotic attractor for $\tau=5.0, a=1.0, b=1.2, c=0.001, p_{1}=0.8, p_{2}=$ 1.33, $x(t)=0.9, t \in(-5,0)$. 
Figure 4: Bifurcation diagrams of single cell DCNN for the parameters values $a=0.16, b=$ 0.2 and $\tau=25.0$ when $p_{2}=1.33$ (a) for transients of the order $1.0-2.0 \times 10^{4}$ and (b) for transients now of the order $1.0 \times 10^{5}$.

Figure 5: Bifurcation diagrams of single cell DCNN for the parameter values $a=0.16, b=$ 0.2 and $\tau=25.0$ when $p_{2}=1.66$ (a) for transients of the order $1.0 \times 10^{5}$ and (b) for transients of order $1.4 \times 10^{6}$.

Figure 6: Bifurcation diagrams of single cell DCNN for the parameter values $a=1.0, b=$ $1.2, c=0.001, p_{2}=1.33$ and $\tau \in(2,29)$ (a) for transients of the order $1.0 \times 10^{5}$ and (b) for transients of the order $2.5 \times 10^{5}$.

Figure 7: Global bifurcation diagrams of single cell DCNN for $\tau \in(0.5,30)$ and $c \in$ $(-0.16,0.16)$. The following colour codes are used to represent various regions, period-1 region -red, period-2 region -green, 3-blue, 4-yellow, 5-magenta, 6-cyan, 7-gray, 8-copper, chaos-black and the fixed points-white. (a) $p_{2}=1.0$, (b) $p_{2}=1.33$ and (c) $p_{2}=1.66$.

Figure 8: The first ten maximal Lyapunov exponents for the values $a=1.0, b=1.2, c=$ $0.001, p_{2}=1.33$ and $\tau \in(2,29)$.

Figure 9: Curves of Eq. (24a) and Eq. (24b) in the range of $-p_{1}<x \leq p_{1}$. The solid curve represents $\tau_{1}$ for $n=0,+1,+2$ and broken curve represents $\tau_{2}$ for $n=+1,+2$. The region enclosed between the line $\tau=0$ and the curve $\tau=\tau_{1}(0,1.5 b)$ is the only stable island (shaded region).

Figure 10: Bifurcation diagrams of coupled cell defined by Eq. (16). (a) For the parameters values $a=0.16, b=0.2, d=0.2, e=0.2$ and $\tau=25.0$ for $p_{2}=1.33$ and for transients of the order $2.0 \times 10^{5}$ and (b) For the same parameter values as in (a) except for the transients of the order $1.75 \times 10^{6}$.

Figure 11: Bifurcation diagrams of the two cell DCNN (a) For the parameters values $a=0.16, b=0.2, d=0.2, e=0.2$ and $\tau=25.0$ when $p_{2}=1.66$ for transients of the order $2.0 \times 10^{5}$ and (b) For the same parameter values as in (a) except for transients of the order $2.0 \times 10^{6}$.

Figure 12: Intermittent behavior at the parameter values $a=0.16, b=0.2, d=0.2, e=0.2$ and $\tau=25.0$ when $p_{2}=1.66$ for critical value of the parameter $c_{\text {crit }}=0.345969$.

Figure 13: Mean laminar length $<l>$ versus $f=c_{\text {crit }}-c$. 
Figure 14: Global bifurcation diagrams of coupled cell DCNN for $\tau \in(0.1,30)$ and $c \in$ $(0.3,1.4)$. The colour codes are the same as in Fig. 7. (a) $p_{2}=1.0$, (b) $p_{2}=1.33$ and (c) $p_{2}=1.66$. 
This figure "fig1.gif" is available in "gif" format from: http://arxiv.org/ps/nlin/0408035v1 
This figure "fig2.gif" is available in "gif" format from: http://arxiv.org/ps/nlin/0408035v1 
This figure "fig3.gif" is available in "gif" format from: http://arxiv.org/ps/nlin/0408035v1 
This figure "fig4.gif" is available in "gif" format from: http://arxiv.org/ps/nlin/0408035v1 
This figure "fig5.gif" is available in "gif" format from: http://arxiv.org/ps/nlin/0408035v1 
This figure "fig6.gif" is available in "gif" format from: http://arxiv.org/ps/nlin/0408035v1 
This figure "fig7a.gif" is available in "gif" format from: http://arxiv.org/ps/nlin/0408035v1 
This figure "fig7b.gif" is available in "gif" format from: http://arxiv.org/ps/nlin/0408035v1 
This figure "fig7c.gif" is available in "gif" format from: http://arxiv.org/ps/nlin/0408035v1 
This figure "fig8.gif" is available in "gif" format from: http://arxiv.org/ps/nlin/0408035v1 
This figure "fig9.gif" is available in "gif" format from: http://arxiv.org/ps/nlin/0408035v1 
This figure "fig10.gif" is available in "gif" format from: http://arxiv.org/ps/nlin/0408035v1 
This figure "fig11.gif" is available in "gif" format from: http://arxiv.org/ps/nlin/0408035v1 
This figure "fig12.gif" is available in "gif" format from: http://arxiv.org/ps/nlin/0408035v1 
This figure "fig13.gif" is available in "gif" format from: http://arxiv.org/ps/nlin/0408035v1 
This figure "fig14a.gif" is available in "gif" format from: http://arxiv.org/ps/nlin/0408035v1 
This figure "fig14b.gif" is available in "gif" format from: http://arxiv.org/ps/nlin/0408035v1 
This figure "fig14c.gif" is available in "gif" format from: http://arxiv.org/ps/nlin/0408035v1 